TITLE:

\title{
Thermal conduction in a chain of colliding harmonic oscillators revisited
}

\section{$\operatorname{AUTHOR(S):~}$}

Sano, Mitsusada M.; Kitahara, Kazuo

\section{CITATION:}

Sano, Mitsusada M.... [et al]. Thermal conduction in a chain of colliding harmonic oscillators revisited. Physical Review E 2001, 64(5(Part 2)): 056111.

\section{ISSUE DATE:}

2001-11

URL:

http://hdl.handle.net/2433/59077

RIGHT:

Copyright 2001 American Physical Society 
PHYSICAL REVIEW E, VOLUME 64, 056111

\title{
Thermal conduction in a chain of colliding harmonic oscillators revisited
}

\author{
Mitsusada M. Sano \\ Department of Fundamental Sciences, FIHS, Kyoto University, Sakyo-ku, Kyoto, 606-8501, Japan \\ Kazuo Kitahara \\ Department of Physics, International Christian University, 10-2, Osawa 3-chome, Mitaka-shi, Tokyo, 181-8585, Japan
}

(Received 2 July 2001; published 22 October 2001)

\begin{abstract}
Thermal conduction in a chain of colliding harmonic oscillators, sometimes called the ding-dong model, is investigated. We first argue that this system is equivalent to the Dawson plasma sheet model. Next we show the Lyapunov analysis for this system to characterize its dynamical property. Finally, we reconsider the numerical study of thermal conduction for this system using the Green-Kubo relation and the direct simulation of Fourier law. Both show that thermal conduction is normal in that $\kappa(N, T) \propto N^{0}$, at least, at low temperature in a large system.
\end{abstract}

DOI: 10.1103/PhysRevE.64.056111

PACS number(s): 05.90.+m, 44.10.+i, 05.45.-a, 05.70.Ln

\section{INTRODUCTION}

Thermal conduction in one-dimensional coupled anharmonic oscillators has been one of the central problems in nonlinear science since the pioneer work by Fermi, Pasta, and Ulam (FPU) [1]. The original motivation of the numerical work by FPU was to check equipartition of energy in one-dimensional coupled anharmonic oscillators. If the system is ergodic, equipartition of energy is expected. However, unexpectedly, equipartition was not realized in their simulation. Instead, recurrence of the initial state was observed. This astonishing result led to two directions of further investigation. One is the discovery of solitons or integrable systems. The other is further numerical study of equipartition or thermalization. The historical perspective is well reviewed in Ford's article [2]. In the second direction, a vast number of numerical studies has been done. Mainly they investigated (1) equipartition of energy, (2) local thermal equilibrium, and (3) transport properties, i.e., thermal conduction. Some of these studies showed that some class of systems displays thermal conduction at finite system size, i.e., the Fourier law is observed. However, it is still unknown whether the thermal conductivity in the thermodynamic limit is finite or not. More precisely, what is the necessary and sufficient condition for normal conduction in one-dimensional anharmonic oscillator systems?

Recently these puzzles and confusions have been partially resolved. The key point is that if the heat current has a hydrodynamical mode the autocorrelation function for this mode has a long-time tail. Then, from the Green-Kubo formula, the time integral of the current autocorrelation function diverges. Thus the thermal conductivity diverges in the thermodynamic limit. Numerical evidence was observed in [3] and analyzed by mode-coupling theory in $[4,5]$. Finally, in a general setting for one-dimensional coupled anharmonic oscillators, Prosen and Campbell have shown that momentum conservation implies anomalous thermal conduction, namely, the thermal conductivity of such one-dimensional systems diverges in the thermodynamic limit [6]. On the other hand, coupled anharmonic oscillators with on-site potential do not satisfy momentum conservation. It is still controversial whether such systems have normal transport coefficients.

In [7], a chain of colliding harmonic oscillators, sometimes called the ding-dong model, was investigated. The Hamiltonian of this system is given by

$$
\mathcal{H}=\sum_{i=1}^{N} \frac{p_{i}^{2}}{2 m}+\frac{1}{2} m \omega_{p}^{2} q_{i}^{2},
$$

with the elastic constraints

$$
q_{i}-q_{i+1} \leqslant \Delta \quad(i=1,2, \ldots, N-1) .
$$

$q_{i}$ represents the displacement of the $i$ th oscillator from its equilibrium point. $\Delta$ is the lattice spacing. This system represents coupled ahnarmonic oscillators with on-site potential. In [7], thermal conduction in this system was investigated. However, the numerical study was limited to system sizes with $N=10-100$. In a similar model, the ding-a-ling model [8], saturation of system-size dependent thermal conductivity evaluated from direct observation of the Fourier law starts around the system size $N=300$ [9]. This observation suggests that we have to take the system size sufficiently large. We believe that the analysis in [7] is insufficient for determining the system-size dependence of the thermal conductivity of the ding-dong model. As another aspect of statistical behavior of this system, it has been shown numerically in $[10]$ that its stationary state in heat conduction satisfies the fluctuation theorem.

In addition, the ding-dong model is equivalent to the Dawson plasma sheet model [11] whose Hamiltonian is given by

$$
\begin{aligned}
\mathcal{H}= & \sum_{i=1}^{N} \frac{P_{i}^{2}}{2 m}-\frac{2 \pi \sigma^{2}}{\varepsilon_{0}} \sum_{i<j}\left|Q_{i}-Q_{j}\right| \\
& +\frac{2 \pi \sigma^{2}}{\varepsilon_{0}}\left(\frac{N}{L}\right) \sum_{i=1}^{N} \int_{0}^{L} d Q\left|Q-Q_{i}\right| \\
& -\frac{\pi \sigma^{2}}{\varepsilon_{0}}\left(\frac{N}{L}\right)^{2} \int_{0}^{L} d Q \int_{0}^{L} d Q^{\prime}\left|Q-Q^{\prime}\right|,
\end{aligned}
$$


where $\varepsilon_{0}$ is the dielectric constant for the vacuum. The equation of motion is

$$
\frac{d^{2}}{d t^{2}} Q_{i}=-\omega_{p}^{2}\left(Q_{i}-\Delta \sum_{j=1}^{N} \theta\left(Q_{i}-Q_{j}\right)\right),
$$

with the plasma frequency $\omega_{p}^{2}=4 \pi \sigma^{2} n / m \varepsilon_{0}$ and the mean spacing of sheets $\Delta=L / N=1 / n$. The step function $\theta(x)$ is defined as

$$
\theta(x)= \begin{cases}1, & x>0 \\ \frac{1}{2}, & x=0 \\ 0, & x<0 .\end{cases}
$$

As we can see from the equation of motion Eq. (4), the difference between the ding-dong model and the Dawson plasma sheet model is whether two particles collide with hard-core interaction or pass through each other. Therefore as far as energy transport is concerned the two systems are equivalent. However, this equivalence holds only in the case of the free boundary condition. For the case of the periodic boundary condition, this equivalence no longer holds [12].

Returning to the problem of the system-size dependence of thermal conductivity, let us consider applying the method in [6] to the ding-dong model. For the ding-dong model, there is no momentum conservation, but the system has two conserved quantities. One is the total energy, and the other is the energy of motion of the center of mass,

$$
C=\frac{1}{2 m}\left(\sum_{i=1}^{N} p_{i}\right)^{2}+\frac{1}{2} m \omega_{p}^{2}\left(\sum_{i=1}^{N} q_{i}\right)^{2},
$$

which is an even function of the total momentum. Thus, the key term in the method of [6] vanishes. Then the method of [6] for this extra conserved quantity yields a trivial inequality $\kappa \geqslant 0$, where $\kappa$ is the thermal conductivity in the thermodynamic limit. So at present the question about the thermal conductivity of the ding-dong model in the thermodynamic limit is still open. Detailed reexamination of thermal conduction in the ding-dong model is therefore needed. This is one of the aims of the present paper.

After describing the dynamics of our system in Sec. II first, we give the Lyapunov analysis of this system in Sec. III. This enables us to characterize its dynamical properties. As a result, from the behavior of the Lyapunov spectrum, we know that the ding-dong model is a weakly chaotic (in other words, mixed) system. Next we carry out a detailed numerical study of thermal conduction in the ding-dong model in Sec. IV. We have evaluated thermal conductivity by two methods. One method uses the Green-Kubo relation. Thus, we evaluate the thermal conductivity from the equilibrium properties (without thermal reservoir). The other method is direct observation of the Fourier law. We observe the nonequilibirum stationary state of a given finite system and evaluate the thermal conductivity from the heat current and the temperature profile in the stationary state. These two methods show that the thermal conductivity in the thermo- dynamic limit seems to be constant, at least at low temperature. In Sec. V, we summarize our results.

\section{MODEL}

As for the system of falling balls treated in [13], it will be convenient for us to construct the associated map, which is defined among collisions. The positions and momenta of all particles are represented by the vector $\mathbf{x}$ :

$$
\mathbf{x}=\left(\begin{array}{c}
q_{1} \\
\vdots \\
q_{N} \\
p_{1} \\
\vdots \\
p_{N}
\end{array}\right) .
$$

We denote the state just after the $k$ th collision by $\mathbf{x}_{k}^{+}$and the state just before the $k$ th collision by $\mathbf{x}_{k}^{-}$. The time when the $k$ th collision takes place between the $i_{k}$ th particle and the $\left(i_{k}+1\right)$ th particle is denoted by $t_{k}$. We take $t_{0}=0$. Our system is formally considered as the dynamical system

$$
\frac{d}{d t} \mathbf{x}=\mathbf{F}(\mathbf{x}), \quad \mathbf{x} \in K,
$$

where $K$ is defined by

$$
K=\left\{\mathbf{x} \in \mathbb{R}^{N} \times \mathbb{R}^{N} \mid \mathcal{H}=E, C=C_{0}\right\} .
$$

Here $E$ and $C_{0}$ are some constants. The flow $\phi^{t}$ defined by Eq. (8) is given by

$$
\phi^{t}: K \rightarrow K \text {. }
$$

Now we define a manifold

$$
M=\left\{\mathbf{x} \in K \mid q_{i}-q_{i+1}<\Delta \text { for } i=1, \ldots, N-1\right\} \subset K .
$$

$M$ is an open subset with compact closure $\bar{M}$ and it has the piecewise smooth boundary $\partial M . \partial M$ is split into the regular part $\partial M_{r}$ and the singular part $\partial M_{s}$. The regular part corresponds to two-body collisions, while the singular part corresponds to collisions involving more than two bodies and collisions of more than one pair at the same time. The regular part $\partial M_{r}$ of the boundary of $M$ is the union of $(N-1)$ submanifolds,

$$
\partial M_{r}=\bigcup_{i=1}^{N-1} \partial M_{i},
$$

where

$$
\begin{aligned}
\partial M_{1} & =\left\{\mathbf{x} \in K \mid q_{1}-q_{2}=\Delta, q_{2}-q_{3}<\Delta, \ldots, q_{N-1}-q_{N}\right. \\
& <\Delta\},
\end{aligned}
$$




$$
\begin{aligned}
\partial M_{2}= & \left\{\mathbf{x} \in K \mid q_{1}-q_{2}<\Delta,\right. \\
<\Delta\}, & q_{2}-q_{3}=\Delta, \ldots, q_{N-1}-q_{N} \\
& \vdots \\
\partial M_{N-1} & =\left\{\mathbf{x} \in K \mid q_{1}-q_{2}<\Delta, q_{2}-q_{3}<\Delta, \ldots, q_{N-1}-q_{N}\right. \\
& =\Delta\} .
\end{aligned}
$$

The regular part $\partial M_{r}$ further splits into $\partial M_{r}^{+}=\cup_{i=1}^{N-1} \partial M_{i}^{+}$ and $\partial M_{r}^{-}=\cup_{i=1}^{N-1} \partial M_{i}^{-}$where

$$
\begin{aligned}
\partial M_{1}^{ \pm}= & \left\{\mathbf{x} \in K \mid q_{1}-q_{2}=\Delta, q_{2}-q_{3}<\Delta, \ldots, q_{N-1}-q_{N}\right. \\
& \left.<\Delta, \mp\left(p_{1}-p_{2}\right)>0\right\}, \\
\partial M_{2}^{ \pm}= & \left\{\mathbf{x} \in K \mid q_{1}-q_{2}<\Delta, q_{2}-q_{3}=\Delta, \ldots, q_{N-1}-q_{N}<\Delta,\right. \\
& \left.\mp\left(p_{2}-p_{3}\right)>0\right\}, \\
\partial M_{N-1}^{ \pm} & =\left\{\mathbf{x} \in K \mid q_{1}-q_{2}<\Delta, q_{2}-q_{3}<\Delta, \ldots, q_{N-1}-q_{N}\right. \\
& \left.=\Delta, \mp\left(p_{N-1}-p_{N}\right)>0\right\} .
\end{aligned}
$$

In $\partial M_{r}^{+} F$ points inside, while in $\partial M_{r}^{-} F$ points outside. That is, $\partial M_{r}^{+}$corresponds to states immediately after collisions and $\partial M_{r}^{-}$to states immediately before collisions.

Here we consider only two-body collisions and disregard collisions involving more than two bodies and collisions of more than one pair, since it is expected that these collisions are events of zero measure.

Now we define the collision map $\Phi_{c o l}: \partial M_{r}^{-} \rightarrow \partial M_{r}^{+}$. More specifically, we define the collision map for the $i$ th and the $(i+1)$ th particles $\Phi_{c o l}^{(i)}: \partial M_{i}^{-} \rightarrow \partial M_{i}^{+}$. Next we define the map for the smooth flow $\Phi_{o s c}^{\tau(\mathbf{x})}: M \cup \partial M_{r}^{+} \rightarrow \partial M_{r}^{-}$for $\mathbf{x}$ $\in M \cup \partial M_{r}^{+}$, where $\tau(\mathbf{x})$ is the time that it takes from the state $\mathbf{x}$ to the next collision. $\tau(\mathbf{x})$ is called the first collision time. More specifically, if the $k$ th collision occurs between the $i_{k}$ th and $\left(i_{k}+1\right)$ th particles, then we may write $\Phi_{o s c}^{\tau\left(\mathbf{x}_{k}^{+}\right)}$: $\partial M_{i_{k}}^{+} \rightarrow \partial M_{i_{k+1}}^{-}$. By definition, the time between successive collisions is given by

$$
\tau\left(\mathbf{x}_{k}^{+}\right)=t_{k+1}-t_{k} .
$$

With this setup, we now define the map $\Phi_{k}$ that maps the state $\mathbf{x}_{k}^{+}$just after the $k$ th collision to the state $\mathbf{x}_{k+1}^{+}$just after the $(k+1)$ th collision. $\Phi_{k}$ is decomposed into two parts, the motion of the independent harmonic oscillators and the collision process,

$$
\Phi_{k}=\Phi_{c o l}^{\left(i_{k+1}\right)} \circ \Phi_{o s c}^{\tau\left(\mathbf{x}_{k}^{+}\right)}
$$

Obviously, we have

$$
\Phi_{k}: \quad \partial M_{i_{k}}^{+} \rightarrow \partial M_{i_{k+1}}^{+}
$$

Succesive iteration gives the following relation:

$$
\mathbf{x}_{n}^{+}=\Phi_{n-1} \circ \Phi_{n-2^{\circ}} \cdots \circ \Phi_{0}\left(\mathbf{x}_{0}^{+}\right)
$$

and

$$
\begin{gathered}
\Phi_{n-1} \circ \Phi_{n-2} \circ \cdots \circ \Phi_{0}: \quad \partial M_{i_{0}}^{+} \rightarrow \partial M_{i_{1}}^{+} \rightarrow \cdots \rightarrow \partial M_{i_{n-2}}^{+} \\
\rightarrow \partial M_{i_{n-1}}^{+} \rightarrow \partial M_{i_{n}}^{+}
\end{gathered}
$$

Next we give the matrix representation of these maps. At each collision, the colliding particles just exchange their momenta. Therefore, the map of the collision process is given in the following way. Suppose that at the $k$ th collision, the $i_{k}$ th particle and the $\left(i_{k}+1\right)$ th particle collide with each other. The map from the state $\mathbf{x}_{k}^{-}$to the state $\mathbf{x}_{k}^{+}$is represented by the matrix

$$
\mathcal{M}_{c o l}^{\left(i_{k}\right)}=\left(\begin{array}{ll}
I_{N} & 0 \\
0 & C_{i_{k}}
\end{array}\right),
$$

where $I_{N}$ is the $N \times N$ identity matrix and

$$
C_{i_{k}}=\left(\begin{array}{cccccccc} 
& & & i_{k} \text { th } & & & & \\
1 & & & \downarrow & & & & \\
& \ddots & & & & & & \\
& & 1 & & & & & \\
& & & 0 & 1 & & & \leftarrow i_{k} \text { th } \\
& & & 1 & 0 & & & \\
& & & & & 1 & & \\
& & & & & & \ddots & \\
& & & & & & & 1
\end{array}\right),
$$

where vacant entries represent zero. Thus, we have

$$
\mathbf{x}_{k}^{+}=\mathcal{M}_{c o l}^{\left(i_{k}\right)} \mathbf{x}_{k}^{-}
$$

The motion of the independent harmonic oscillators is easily constructed. Set the state $\mathbf{x}_{k}^{+}$just after the $k$ th collision and the state $\mathbf{x}_{k+1}^{-}$just before the $(k+1)$ th collision. The map between these states is

$$
\mathbf{x}_{k+1}^{-}=\mathcal{M}_{o s c}^{\tau\left(\mathbf{x}_{k}^{+}\right)} \mathbf{x}_{k}^{+} .
$$

The matrix $\mathcal{M}_{o s c}^{\tau\left(\mathbf{x}_{k}^{+}\right)}$is given by 


$$
\begin{aligned}
& \mathcal{M}_{\text {osc }}^{\tau\left(\mathbf{x}_{k}^{+}\right)} \\
& \quad=\left(\begin{array}{ll}
\cos \left[\omega_{p} \tau\left(\mathbf{x}_{k}^{+}\right)\right] I_{N} & \frac{1}{m \omega_{p}} \sin \left[\omega_{p} \tau\left(\mathbf{x}_{k}^{+}\right)\right] I_{N} \\
-m \omega_{p} \sin \left[\omega_{p} \tau\left(\mathbf{x}_{k}^{+}\right)\right] I_{N} & \cos \left[\omega_{p} \tau\left(\mathbf{x}_{k}^{+}\right)\right] I_{N}
\end{array}\right) .
\end{aligned}
$$

Thus we have obtained the matrix representation of the whole time evolution,

$$
\mathbf{x}_{n}^{+}=\prod_{k=0}^{n-1}\left(\mathcal{M}_{c o l}^{\left(i_{k+1}\right)} \mathcal{M}_{\text {osc }}^{\tau\left(\mathbf{x}_{k}^{+}\right)}\right) \mathbf{x}_{0}^{+},
$$

where the product is time ordered.

\section{LYAPUNOV ANALYSIS}

The dynamics of this system is singular at collisions. Thus, the Lyapunov analysis differs from those for smooth dynamical systems. We have to reformulate the Lyapunov analysis in a suitable form. In order to get the tangent map of the map obtained in the previous section, we change the variables from Cartesian coordinates to the action-angle variables

$$
q_{i}=\sqrt{\frac{2 J_{i}}{m \omega_{p}}} \cos \left(\alpha_{i}\right), \quad p_{i}=\sqrt{2 m \omega_{p} J_{i}} \sin \left(\alpha_{i}\right),
$$

for $i=1,2, \ldots, N$. The Hamiltonian becomes

$$
\mathcal{H}=\omega_{p} \sum_{i=1}^{N} J_{i}+(\text { hard-core collisions }) .
$$

Between the collisions, the equation of motion is given by

$$
\frac{d}{d t} J_{i}=0 \quad \text { and } \quad \frac{d}{d t} \alpha_{i}=\omega_{p} .
$$

Therefore, we have

$$
J_{i}(t)=\mathrm{const} \quad \text { and } \quad \alpha_{i}(t)=\omega_{p} t+\alpha_{i}(0) .
$$

Now we define the new vector $\mathbf{X}$,

$$
\mathbf{X} \equiv\left(\begin{array}{c}
J_{1} \\
\vdots \\
J_{N} \\
\alpha_{1} \\
\vdots \\
\alpha_{N}
\end{array}\right) .
$$

The equation of motion between collisions is now

$$
\frac{d}{d t} \mathbf{X}=-J \nabla_{\mathbf{X}} \mathcal{H}
$$

where $J$ is the symplectic matrix

$$
J=\left(\begin{array}{cc}
0 & I_{N} \\
-I_{N} & 0
\end{array}\right)
$$

Between collisions, the time evolution of the infinitesimal deviation $\delta \mathbf{X}$ is given by

$$
\frac{d}{d t} \delta \mathbf{X}=\left(\begin{array}{cc}
-\frac{\partial^{2} \mathcal{H}}{\partial J \partial \alpha} & -\frac{\partial^{2} \mathcal{H}}{\partial \alpha^{2}} \\
\frac{\partial^{2} \mathcal{H}}{\partial J^{2}} & \frac{\partial^{2} \mathcal{H}}{\partial \alpha \partial J}
\end{array}\right) \delta \mathbf{X}=\left(\begin{array}{ll}
0 & 0 \\
0 & 0
\end{array}\right) \delta \mathbf{X} .
$$

Thus, between the collisions, the time evolution of $\delta \mathbf{X}$ is just

$$
\delta \mathbf{X}(t)=\delta \mathbf{X}(0),
$$

as long as no collisions occur. Therefore, in order to get the tangent map, we have to consider only the contribution from the collisions.

Let us consider how to change $\delta \mathbf{X}$ in the collision process. We denote the infinitesimal quantities after and before the $k$ th collision by $\delta \mathbf{X}_{k}^{-}$and $\delta \mathbf{X}_{k}^{+}$, respectively. What we need is the $2 N \times 2 N$ monodromy matrix $M_{k}$ satisfying the following condition:

$$
\delta \mathbf{X}_{k}^{+}=M_{k} \delta \mathbf{X}_{k}^{-} .
$$

Here we note the relation among $\delta \mathbf{X}_{k}^{ \pm}$and the $\delta \mathbf{x}_{k}^{ \pm}$'s. From Eq. (26), we obtain the relations

$$
\begin{gathered}
\delta \mathbf{X}_{k}^{+}=R\left(\mathbf{x}_{k}^{+}\right)^{-1} \delta \mathbf{x}_{k}^{+}, \\
\delta \mathbf{x}_{k}^{-}=R\left(\mathbf{x}_{k}^{-}\right) \delta \mathbf{X}_{k}^{-},
\end{gathered}
$$

where

$$
R\left(\mathbf{x}_{k}^{-}\right)=\left(\begin{array}{ll}
R_{11} & R_{12} \\
R_{21} & R_{22}
\end{array}\right)
$$

$$
R\left(\mathbf{x}_{k}^{+}\right)^{-1}=\left(\begin{array}{ll}
Q_{11} & Q_{12} \\
Q_{21} & Q_{22}
\end{array}\right)
$$

with the matrices

$$
R_{11}=\left(\begin{array}{ccc}
\frac{q_{1}^{-}}{2 J_{1}^{-}} & & \\
& \ddots & \\
& & \frac{q_{N}^{-}}{2 J_{N}^{-}}
\end{array}\right)
$$

$$
R_{12}=\left(\begin{array}{ccc}
-\frac{p_{1}^{-}}{m \omega_{p}} & & \\
& \ddots & \\
& & -\frac{p_{N}^{-}}{m \omega_{p}}
\end{array}\right),
$$




$$
\begin{gathered}
R_{21}=\left(\begin{array}{ccc}
\frac{p_{1}^{-}}{2 J_{1}^{-}} & & \\
& \ddots & \\
& & \frac{p_{N}^{-}}{2 J_{N}^{-}}
\end{array}\right), \\
R_{22}=\left(\begin{array}{lll}
m \omega_{p} q_{1}^{-} & & \\
& \ddots & \\
& & m \omega_{p} q_{N}^{-}
\end{array}\right),
\end{gathered}
$$

and

$$
\begin{gathered}
Q_{11}=\left(\begin{array}{ccc}
m \omega_{p} q_{1}^{+} & & \\
& \ddots & \\
& & m \omega_{p} q_{N}^{+}
\end{array}\right), \\
Q_{12}=\left(\begin{array}{ccc}
\frac{p_{1}^{+}}{m \omega_{p}} & & \\
& \ddots & \\
& & \frac{p_{N}^{+}}{m \omega_{p}}
\end{array}\right), \\
Q_{21}=\left(\begin{array}{ccc}
\frac{p_{1}^{+}}{2 J_{1}^{+}} & & \\
& \ddots & \\
& & -\frac{p_{N}^{+}}{2 J_{N}^{+}}
\end{array}\right), \\
Q_{22}=\left(\begin{array}{ccc}
\frac{q_{1}^{+}}{2 J_{1}^{+}} & & \\
& \ddots & \\
& & \frac{q_{N}^{+}}{2 J_{N}^{+}}
\end{array}\right) .
\end{gathered}
$$

In the $R_{i j}$ and $Q_{i j}$ all vacant entries represent zero. At the $k$ th collision, the $i_{k}$ th particle and $\left(i_{k}+1\right)$ th particle exchange their momenta. In addition, the condition $q_{i_{k}}-q_{i_{k}+1}=\Delta$ is satisfied. Therefore, the infinitesimal quantities satisfy $\delta q_{i_{k}}$ $=\delta q_{i_{k}+1}$. Using this condition, we obtain the relation between $\delta \mathbf{x}_{k}^{-}$and $\delta \mathbf{x}_{k}^{+}$:

$$
\delta \mathbf{x}_{k}^{+}=N_{k} \delta \mathbf{x}_{k}^{-},
$$

where

$$
N_{k}=\left(\begin{array}{ll}
C_{i_{k}} & 0 \\
0 & C_{i_{k}}
\end{array}\right) .
$$

To set $N_{k}$ in this way is important for the symplectic condition of the tangent map. Now, using Eqs. (36), (37), and (44), we have

$$
\begin{aligned}
\delta \mathbf{X}_{k}^{+} & =R\left(\mathbf{x}_{k}^{+}\right)^{-1} \delta \mathbf{x}_{k}^{+}=R\left(\mathbf{x}_{k}^{+}\right)^{-1} N_{k} \delta \mathbf{x}_{k}^{-} \\
& =R\left(\mathbf{x}_{k}^{+}\right)^{-1} N_{k} R\left(\mathbf{x}_{k}^{-}\right) \delta \mathbf{X}_{k}^{-} .
\end{aligned}
$$

Thus, $M_{k}$ is given by

$$
M_{k}=R\left(\mathbf{x}_{k}^{+}\right)^{-1} N_{k} R\left(\mathbf{x}_{k}^{-}\right) .
$$

The matrices $R\left(\mathbf{x}_{k}^{+}\right)^{-1}, N_{k}$, and $R\left(\mathbf{x}_{k}^{-}\right)$obtained here satisfy the symplectic condition

$$
{ }^{t} M J M=J \quad \text { where } \quad M=R\left(\mathbf{x}_{k}^{+}\right)^{-1}, N_{k}, R\left(\mathbf{x}_{k}^{-}\right) .
$$

Therefore, $M_{k}$ itself satisfies the symplectic condition. Finally, the time evolution of the infinitesimal quantity $\delta \mathbf{X}$, namely, the tangent map, is given by

$$
\delta \mathbf{X}_{k}^{-}=M_{k-1} M_{k-2} \cdots M_{1} M_{0} \delta \mathbf{X}_{0}^{-} .
$$

Let us consider the Lyapunov spectrum of this system. First check the dimension of the Lyapunov spectrum. The number of particles is $N$. Then the dimension of the phase space is $2 N$, from which we have to subtract the number of conserved quantities. (1) The total energy $E$ is conserved. (2) The energy of the center of mass is conserved. Thus, the dimension of the Lyapunov spectrum is $2 N-2$. The Lyapunov exponents are given by

$$
\lambda_{m}=\lim _{k \rightarrow \infty} \frac{1}{t_{k}} \ln \left[\frac{\left|\prod_{l=0}^{k-1} M_{l} \delta \mathbf{X}_{0}^{-,(m)}\right|}{\left|\delta \mathbf{X}_{0}^{-,(m)}\right|}\right],
$$

where $\delta \mathbf{X}_{0}^{-,(m)}$ is the displacement to the $m$ th eigendirection and the product of the matrices $M_{i}$ is time ordered. The Lyapunov spectrum is now

$$
\lambda_{1} \geqslant \lambda_{2} \geqslant \cdots \geqslant \lambda_{2 N} .
$$

We have to eliminate two irrelevant Lyapunov exponents which correspond to the above-mentioned conserved quantities. These Lyapunov expoents have zero value. After renumbering, we have the Lyapunov spectrum

$$
\lambda_{1} \geqslant \lambda_{2} \geqslant \cdots \geqslant \lambda_{2 N-2} \text {. }
$$

Here we do not consider the case of a quadruplet, in which the value of the Lyapunov exponents is a complex number. In our case, the Lyapunov exponents make pairs, $\lambda_{1}=$ $-\lambda_{2 N-2}, \lambda_{2}=-\lambda_{2 N-3}, \ldots$. This is a consequence of the symplecticity. Thus, we have

$$
\sum_{m=1}^{2 N-2} \lambda_{m}=0
$$

The numerical result of the calculation of the Lyapunov spectrum for $N=50$ is shown in Fig. 1. It is known from a vast number of numerical studies that for a hyperbolic sys- 


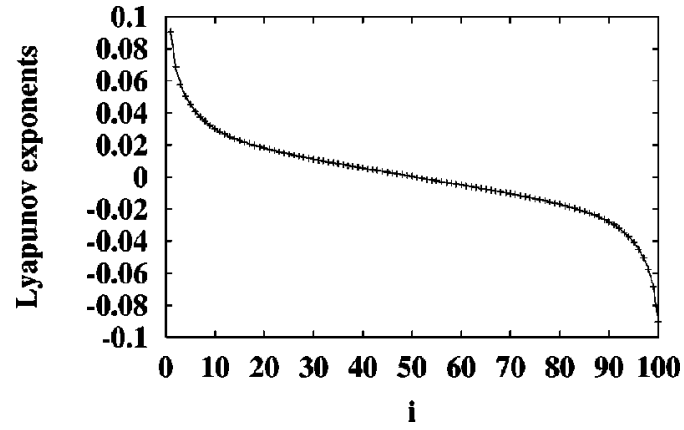

FIG. 1. Lyapunov spectrum: $N=50, E / N=1.0$. The number of samples for averaging is 1000 .

tem, if the Lyapunov spectrum is arranged in decreasing order of indices, it is linear with respect to the indices. However, in Fig. 1, the Lyapunov spectrum is not linear with respect to the indices. This is a typical behavior of mixed systems as shown for various systems in [14]. Thus, Fig. 1 is numerical evidence of the coexistence of stable and unstable motions. Next we consider the Kolmogorov-Sinai (KS) entropy $h_{K S}$ which is a characteristic quantity for the chaoticity of a given system. Since the ding-dong model does not seem to be a hyperbolic system as shown in Fig. $1, h_{K S}$ is not well defined for the ding-dong model. Therefore we define $h_{K S}$ tentatively by the Pesin formula, which is valid only for hyperbolic systems,

$$
h_{K S}=\sum_{\lambda_{i}>0} \lambda_{i}
$$

We show the behavior of $h_{K S}$ versus $E / N$ in Fig. 2 for $N$ $=10$. For small $E / N, h_{K S}$ increases approximately linearly with increasing $E / N$. For $E / N>5 h_{K S}$ increases with increasing $E / N$ very slowly. The fitting (dashed line) is $0.275 \ln (E / N)$.

\section{THERMAL CONDUCTION}

In this section, we study thermal conduction in the dingdong model. The thermal conduction in systems with on-site potential is investigated in $[8,9]$ for the ding-a-ling model and in [7] for the ding-dong model. It is believed that in these models the thermal conduction is normal, that is, the

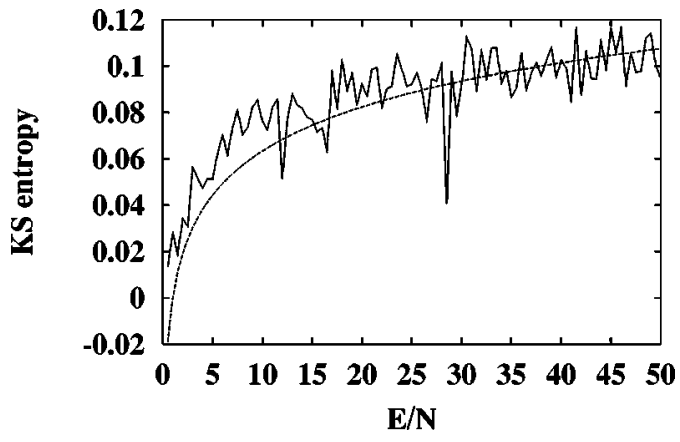

FIG. 2. Kolmogorov-Sinai entropy $h_{K S}$ versus $E / N: N=10$. The number of samples for averaging is 300 . The fitting (dashed line) is $0.0275 \ln (E / N)$. thermal conductivity behaves as $\kappa(N) \propto N^{0}$. However, the authors in [7] commented on the system-size dependence of the thermal conductivity from the numerical results of a system with $N=10-100$ only. There is no systematic study of the system-size dependence of thermal conductivity. The aim of this section is to check this for the ding-dong model. While the thermal conductivity was measured by three different methods for the ding-dong model in [7], we use two methods, (1) the estimation of the thermal conductivity from the Green-Kubo formula and (2) the direct observation of the Fourier law. All discussion in this section will be done by setting $\Delta=m=\omega_{p}=1$.

\section{A. Green-Kubo formalism}

We define the heat current at each site by

$$
\begin{aligned}
j_{k}(t) & =\frac{1}{2}\left(p_{k+1}-p_{k}\right)\left(p_{k+1}^{2}-p_{k}^{2}\right) \delta\left(q_{k}-q_{k+1}-1\right) \\
& =\frac{1}{2}\left(p_{k+1}^{2}-p_{k}^{2}\right) \sum_{n(k)=-\infty}^{\infty} \delta\left(t-t_{n(k)}\right),
\end{aligned}
$$

where $t_{n(k)}$ denotes the $n(k)$ th collision between the $k$ th and $(k+1)$ th sites and the momenta in Eq. (55) should be evaluated just before each collision. We define the total heat current

$$
J_{N}(t)=\sum_{k=1}^{N} j_{k}(t)
$$

and the average heat current over all sites

$$
\bar{J}_{N}(t)=\frac{1}{N} \sum_{k=1}^{N} j_{k}(t) .
$$

The Green-Kubo formula for thermal conduction gives the thermal conductivity as

$$
\kappa(N, T)=\frac{1}{T^{2} N} \int_{0}^{\infty} d \tau\left\langle J_{N}(0) J_{N}(\tau)\right\rangle_{c} .
$$

Here $\langle\cdots\rangle_{c}$ represents the average over the canonical ensemble. If there is no phase transition [15], we can replace this canonical ensemble average by the microcanonical average $\langle\cdots\rangle_{m c}$ specifying the energy from a given temperature,

$$
\langle A\rangle_{c}=\langle A\rangle_{m c}+o\left(\frac{1}{N}\right)
$$

where $A$ is some observable.

In the escape rate formalism for the thermal conduction [16], the following quantity $G_{N}(t)$ plays a central role:

$$
G_{N}(t)-G_{N}(0)=\int_{0}^{t} d \tau \bar{J}_{N}(\tau)
$$




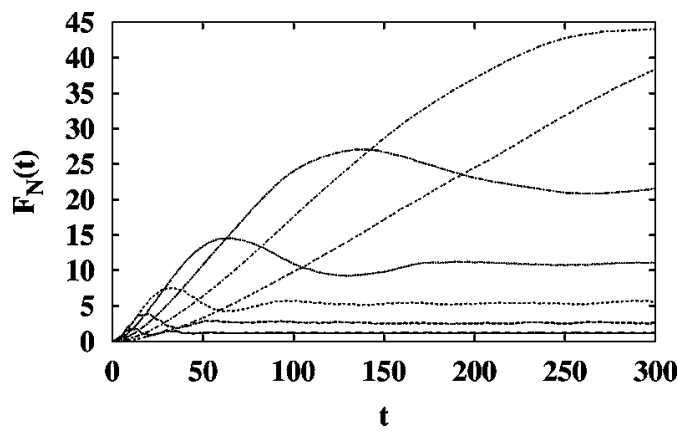

FIG. 3. Behavior of $F_{N}(t): E / N=1.0$. From bottom to top, $N$ $=10,20,40,80,160,320,640$. Each curve is obtained from an average over 5000 initial conditions. In the short time regime, $F_{N}(t)$ diffuses as $F_{N}(t) \sim t$. Then later it converges to some oscillatory value. As the system size becomes larger, the diffusive regime becomes longer. The slope of the diffusive regime is approximately constant. However, there is a tendency to decreasing slope as $n$ the system size increases.

The moment of this quantity is called the Helfand moment [16]. From the diffusive behavior of $G_{N}(t)$ we can discuss thermal conduction. If the diffusive behavior of $G_{N}(t)$ persists forever, we have

$$
\lim _{t \rightarrow \infty} \frac{\left\langle\left[G_{N}(t)-G_{N}(0)\right]^{2}\right\rangle_{m c}}{2 t}=\int_{0}^{\infty} d \tau\left\langle\bar{J}_{N}(0) \bar{J}_{N}(\tau)\right\rangle_{m c}
$$

We now set

$$
F_{N}(t) \equiv \frac{\left\langle\left[G_{N}(t)-G_{N}(0)\right]^{2}\right\rangle_{m c}}{2}
$$

However, in general for a finite system, the diffusive behavior of $G_{N}(t)$ does not persist, but saturates in some time interval, say $t^{*}$. In Fig. 3, we plot the behavior of $F_{N}(t)$ for $E / N=1.0$ and $N=10,20,40,80,160,320$. In the short time regime $t<t^{*}, G_{N}(t)$ diffuses as $F_{N}(t) \sim t$. Then for $t$ $>t^{*} F_{N}(t)$ converges oscillatorily to some value. $t^{*}$ is the time at which the system reaches equilibrium. For larger system size $N, t^{*}$ becomes longer, approximately in proportion to $N$. As the system size becomes large, the slope of $F_{N}(t)$ is approximately constant. There is a tendency to decreasing slope, as the system size increases. This behavior is examined later in detail. We define the diffusion coefficient for finite systems,

$$
D_{N}=\lim _{t \rightarrow t^{*}} \frac{F_{N}(t)}{t}
$$

Here we define the thermal conductivity for a finite system as follows:

$$
\kappa(N, T)=\frac{N}{T^{2}} D_{N}
$$

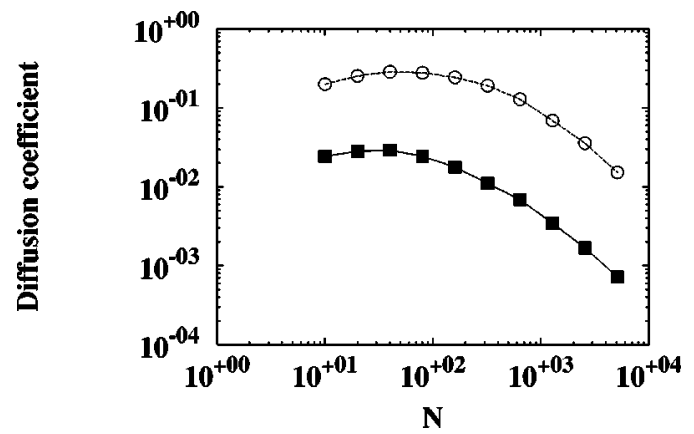

FIG. 4. $N$ dependence of $D_{N}: E / N=0.5$ (solid squares), 1.0 (circles). The number of samples for averaging is 500-1000.

We have to consider the thermodynamic limit $N \rightarrow \infty$. From these observations, the thermal conductivity in the thermodynamic limit would be defined by

$$
\kappa(T)=\frac{1}{T^{2}} \lim _{t \rightarrow \infty} \lim N \frac{F_{N}(t)}{t} .
$$

In Fig. 4, we depict the $N$ dependence of $D_{N}$ for $E / N$ $=0.5,1.0$. This shows $D_{N} \propto N^{-1.0}$. In Fig. 5, we show the result for the $N$ dependece of $N D_{N}$. From Eq. (65), Fig. 3, Fig. 4, and Fig. 5, we know that the thermal conductivity is finite in the thermodynamic limit, at least at low temperature [17]. Of course, we also have to check whether the thermal conductivity $\kappa(T)$ diverges or is finite in the thermodynamic limit by direct numerical simulation of the Fourier law.

In Fig. 6, we plot the energy dependence of the diffusion coefficient $D_{N}(T)$. First, we note the relation between the temperature $T$ and the total energy $E$. From the numerical result, we know

$$
T \approx 1.27 \frac{E}{N}
$$

From the fitting of Fig. 6, we have

$$
D_{N}(T) \propto T^{2.65}
$$

Therefore, the temperature dependence of the thermal conductivity is given by

$$
\kappa(N, T) \propto T^{0.65} \approx T^{2 / 3} .
$$

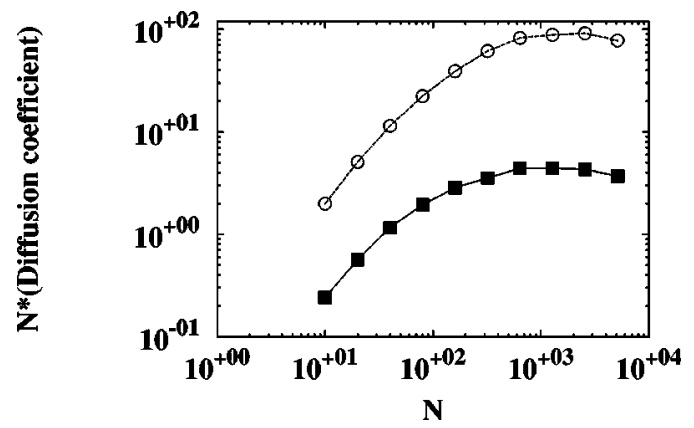

FIG. 5. $N$ dependence of $N D_{N}: E / N=0.5$ (solid squares), 1.0 (circles). The number of samples for averaging is $500-1000$. 


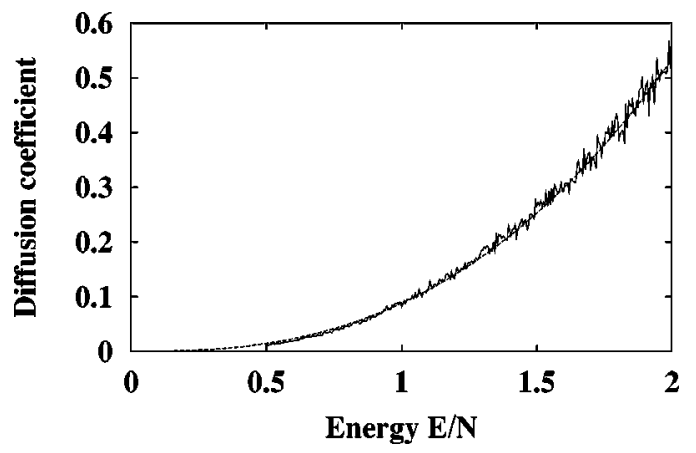

FIG. 6. Temperature dependence of the diffusion coefficient $D_{N}: N=50$. As a result of fitting, it behaves like $D_{N}(T) \sim T^{2.65}$. The number of samples for averaging is 300 .

Next we confirm that the hydrodynamic mode is not important for our system. If the heat current has a hydrodynamic mode, the heat current can be written as

$$
J=J_{d i f f}+n T \delta S v
$$

where $J_{\text {diff }}$ is the heat current due to thermal diffusion, $n$ is the density, $T$ is the temperature, $\delta S$ is the excess entropy, and $v$ is the velocity. The current autocorrelation function can be written as

$$
\langle J(0) J(t)\rangle=\left\langle J_{\text {diff }}(0) J_{\text {diff }}(t)\right\rangle+(n T \delta S)^{2}\langle v(0) v(t)\rangle,
$$

where we assumed that $\left\langle J_{\text {diff }}(0) v(t)\right\rangle=0$ and $\left\langle J_{\text {diff }}(t) v(0)\right\rangle=0$. If the velocity autocorrelation function has a long-time tail, the thermal conductivity will diverge from the Green-Kubo formula. Here we check the velocity autocorrelation function for a tagged particle. In Fig. 7, we depict the power spectrum of the velocity of the tagged particle for the system size $N=200$. The tagged particle is the 100th one from the left. Figure 7 clearly shows that the power spectrum is Lorentzian, not power law. Therefore, in our system, there is no power law behavior in time, at least at low temperature. This implies that there is no hydrodynamic contribution to the heat current. This is another support for the normal thermal conduction in our system.

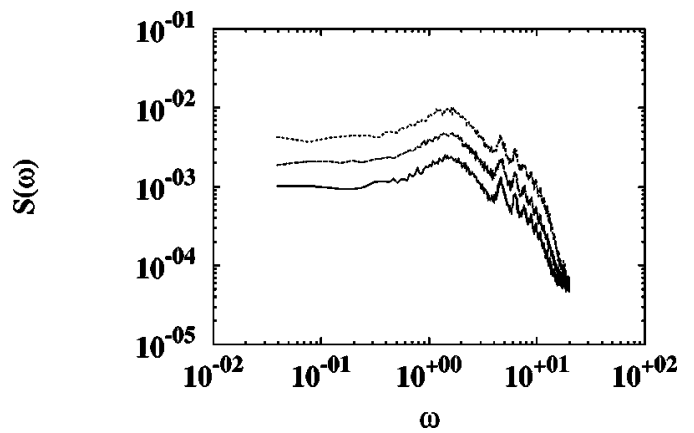

FIG. 7. Power spectrum of velocity of the tagged particle: $N$ $=200$. The tagged particle is the 100th one, namely, the middle particle. From bottom to top, $E / N=0.5,1.0,2.0$. The number of samples for averaging is 250 .

\section{B. Stationary state in a finite system}

For the second method, i.e., the direct observation of the Fourier law, we have to introduce heat reservoirs. We consider two heat reservoirs which consist of ideal gas with temperatures $T_{L}$ (left) and $T_{R}$ (right) and are placed at the left and right sides, respectively. The boundaries between the system and the two reservoirs consist of two walls. The particles in the reservoirs obey the Maxwell distribution at given temperatures. If the particles at the ends of the system, namely, the first particle and the $N$ th particle, collide with the boundary walls, then these particles instantaneously exchange their momenta with the particles of the reservoirs, whose momenta obey the distribution

$$
P(p)=\frac{|p|}{T} \exp \left[-\frac{p^{2}}{T}\right],
$$

where $T=T_{L}\left(T_{R}\right)$ for the first (the $N$ th) particle, respectively. It should be noted that we have to adjust the positions of the walls appropriately so that the particles of the system and the walls may collide efficiently.

The phenomenological Fourier law is given by

$$
\mathcal{J}=-\kappa \nabla T \text {. }
$$

There are two methods to evaluate the thermal conductivity from direct simulation of the stationary state. (1) Thermal conductivity in a global sense is defined by

$$
\kappa_{G}(N, T)=-\mathcal{J}_{N} N /\left(T_{R}-T_{L}\right)
$$

Here $\mathcal{J}_{N}$ is the time average of the energy $\Delta E_{n}$ injected from the (cold and hot) reservoirs,

$$
\mathcal{J}_{N}=\lim _{t \rightarrow \infty} \frac{1}{t} \sum_{n=1}^{m(t)} \Delta E_{n} .
$$

$\Delta E_{n}$ represents the energy exchange with the (cold and hot) reservoirs. $m(t)$ is the number of collisions with the reservoirs up to the time $t$. We may use the heat current averaged over all sites:

$$
\mathcal{J}_{N}=\lim _{t \rightarrow \infty} \frac{1}{t} \int_{0}^{\infty} d \tau \bar{J}_{N}(t)
$$

(2) Thermal conductivity in the local sense is defined as the ratio between the local heat current and the local temperature gradient.

We measure the thermal conductivity in the global sense. In Fig. 8, we depict the $N$ dependence of the thermal conductivity determined by direct observation of the Fourier law. In the high temperature regime, it behaves as $\kappa(N, T)$ $\sim N^{1-\delta}$ where $0<\delta \ll 1$, while in the low temperature regime, it behaves as $\kappa(N, T) \sim N^{0}$, i.e., normal conduction.

\section{SUMMARY}

We have shown that the ding-dong model, unlike the FPU model, indeed exhibits normal conduction, at least in the low temperature regime. This is the central result of this paper. 


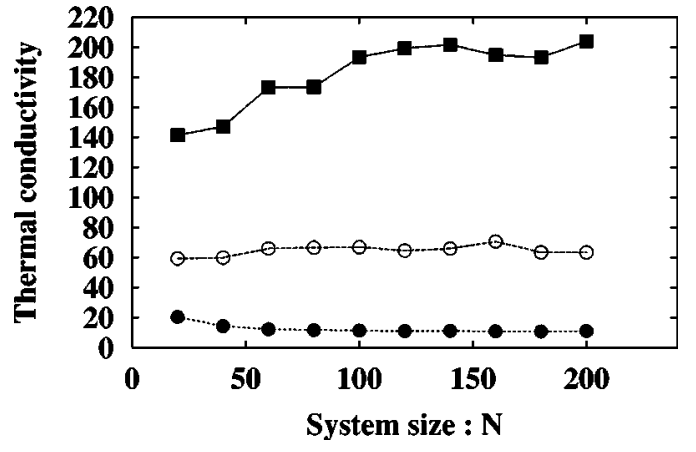

FIG. 8. $N$ dependence of the thermal conductivity by direct observation of the Fourier law: $T_{L}=2.0, T_{R}=1.6$ (solid squares), $T_{L}$ $=1.2, T_{R}=0.8$ (circles), $T_{L}=0.6, T_{R}=0.2$ (solid circles).

As explained in Sec. I, we expect that the Dawson plasma sheet model has the same energy transport properties. The main difference between the FPU model and the ding-dong model is the conserved quantity. For the FPU model the total momentum is conserved, while for the ding-dong model it is not conserved. Instead the ding-dong model has the different conserved quantity $C$ in Eq. (6). The anomalous properties of the conductivity in the FPU model are justified in terms of the conservation of total momentum, as pointed out by Prosen and Campbell [6]. The application of their method to the ding-dong model yields just the trivial inequality $\kappa \geqslant 0$. Therefore, for the ding-dong model there is a regime where the system displays the normal conduction. However, for high temperature, there is the possibility that the ballistic motion destroys the normal conduction. This problem is reserved for future study.

In order for FPU-type models to have normal thermal conduction, the dimension should be larger than 1. In fact, in [20], it is shown that the two-dimensional FPU model still has anomalous conduction, but the three-dimensional FPU model seems to be normal.

Other examples in one dimension that display normal conduction are the hyperbolic billiard system (the Lorentz gas model, a pseudo-one-dimensional system) [18] and the multibaker system with energy, which is a toy model of the Lorentz gas model [19]. These models are independent particle systems. Therefore, these systems are essentially different from the ding-dong model. The ding-dong model is one of the rare examples of one-dimensional interacting particle systems displaying normal conduction. Finally, a kinetic theory for the ding-dong model has been investigated in [21]. It will be published elsewhere.

\section{ACKNOWLEDGMENTS}

We thank Profssor S. Takesue and Professor H. van Beijeren for useful discussions and continuous encouragement. This work was supported by a Grant-in-Aid for Scientific Research (No. 11837018) from the Japan Society for the Promotion of Science. The numerical computation in this work was partially carried out at the Yukawa Institute Computer Facility.
[1] E. Fermi, J. Pasta, and S. Ulam, Collected Papers (University of Chicago Press, Chicago, 1965), Vol. II, p. 978.

[2] J. Ford, Phys. Rep. 213, 271 (1992).

[3] S. Lepri, R. Livi, and A. Politi, Phys. Rev. Lett. 78, 1896 (1997).

[4] S. Lepri, R. Livi, and A. Politi, Europhys. Lett. 43, 271 (1998).

[5] S. Lepri, Phys. Rev. E 58, 7165 (1998).

[6] T. Prosen and D.K. Campbell, Phys. Rev. Lett. 84, 2857 (2000).

[7] T. Prosen and M. Robnik, J. Phys. A 25, 3449 (1992).

[8] G. Casati, J. Ford, F. Vivaldi, and W.M. Visscher, Phys. Rev. Lett. 52, 1861 (1984).

[9] D.J.R. Mimnagh and L.E. Ballentine, Phys. Rev. E 56, 5332 (1997)

[10] M.M. Sano, Phys. Rev. E 61, 1144 (2000).

[11] J.M. Dawson, Phys. Rev. 113, 383 (1959); Phys. Fluids 5, 445 (1962); Methods Comput. Phys. 9, 1 (1970).

[12] M.M. Sano and K. Kitahara (unpublished).
[13] M. Wojtkowski, Commun. Math. Phys. 126, 507 (1990); 127, 425 (1990).

[14] Y.Y. Yamaguchi, J. Phys. A 31, 193 (1998).

[15] This is guaranteed by the following article: H. Kunz, Ann. Phys. (N.Y.) 85, 303 (1974).

[16] P. Gaspard, Chaos, Scattering and Statistical Mechanics (Cambridge University Press, Cambridge, 1998).

[17] Here we assume that the Green-Kubo formula is valid for the corresponding temperature (or energy) regime. Of course, the validity of the Green-Kubo formula should be theoretically confirmed, i.e., the temperature regime and the temperature difference.

[18] D. Alonso, R. Artuso, G. Casati, and I. Guarneri, Phys. Rev. Lett. 82, 1859 (1999).

[19] S. Tasaki and P. Gaspard, J. Stat. Phys. 101, 125 (2000).

[20] T. Shimada, T. Murakami, S. Yukawa, K. Saito, and N. Ito, J. Phys. Soc. Jpn. 69, 3150 (2000).

[21] M.M. Sano and K. Kitahara (unpublished). 